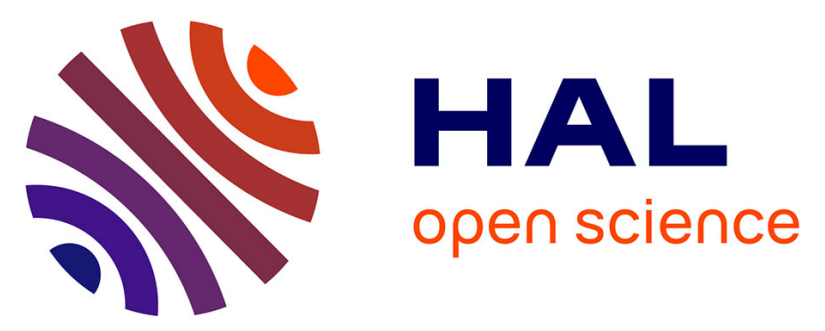

\title{
Guided wave imaging of composite plates using passive acquisitions by fiber Bragg gratings
}

Arnaud Recoquillay, Tom Druet, Simon Nehr, Margaux Horpin, Olivier

Mesnil, Bastien Chapuis, Guillaume Laffont, Oscar d'Almeida

\section{To cite this version:}

Arnaud Recoquillay, Tom Druet, Simon Nehr, Margaux Horpin, Olivier Mesnil, et al.. Guided wave imaging of composite plates using passive acquisitions by fiber Bragg gratings. Journal of the Acoustical Society of America, 2020, 147 (5), pp.3565-3574. 10.1121/10.0001300 cea-02975826

\section{HAL Id: cea-02975826}

https://hal-cea.archives-ouvertes.fr/cea-02975826

Submitted on 23 Oct 2020

HAL is a multi-disciplinary open access archive for the deposit and dissemination of scientific research documents, whether they are published or not. The documents may come from teaching and research institutions in France or abroad, or from public or private research centers.
L'archive ouverte pluridisciplinaire HAL, est destinée au dépôt et à la diffusion de documents scientifiques de niveau recherche, publiés ou non, émanant des établissements d'enseignement et de recherche français ou étrangers, des laboratoires publics ou privés. 


\title{
Guided wave imaging of composite plates using passive acquisitions by fiber Bragg gratings
}

\author{
Arnaud Recoquillay, ${ }^{1, \text { a) }}$ Tom Druet, ${ }^{1}$ Simon Nehr, ${ }^{1}$ Margaux Horpin, ${ }^{1}$ Olivier Mesnil, ${ }^{1}$ Bastien Chapuis, ${ }^{1}$ \\ Guillaume Laffont, ${ }^{1}$ and Oscar D'Almeida ${ }^{2}$ \\ ${ }^{1}$ CEA, LIST, Gif-sur-Yvette, France \\ ${ }^{2}$ Safran Tech, Magny-Les-Hameaux, France
}

In this paper are presented imaging results of defects in composite plates using guided wavebased algorithms such as Delay and Sum and Excitelet. Those algorithms are applied to passive data, for which the signal corresponding to each emitter-receiver couple is recovered thanks to the cross-correlation of the ambient noise measured simultaneously by the two sensors. The transition to passive imaging allow the use of lighter sensors unable to emit ultrasonic waves such as Fiber Bragg Gratings sensors on optical fibers, which are used in this study. The imaging results presented here show the feasibility of active and passive imaging in composite plates using Fiber Bragg Gratings as receivers, reducing the impact of the acquisition system on the structure in the context of Structural Health Monitoring.

(C)2020 Acoustical Society of America. [http://dx.doi.org/10.1121/10.0001300]

$[\mathrm{XYZ}] \quad$ Pages: $1-11$

Keywords: Elastic guided waves, composite plates, Fiber Bragg Gratings, Delay and Sum, Excitelet, passive imaging, Structural Health Monitoring

\section{INTRODUCTION}

Over the last decade, an increasing interest appeared for guided wave-based imaging methods in the context of Non Destructive Testing (NDT) as well as of Structural the inspection of a wide area of a structure with just a few sensors, reducing the inspection time and cost. In particular, the use of less sensors allows to embed them in the structure, making SHM possible. However, SHM systems of a burden for an industrial structure such as an aircraft. Hence the need of reducing this burden, for example by reducing the energy consumption of the sensors or their weight. To do so, passive acquisition methods seem a promising solution: they consist in recovering the impulse response of the system composed of the medium and the sensors by treating the ambient noise measured simultaneously at all sensors. The most common technique is the noise cross-correlation (Lobkis and Weaver, 2001), which used succesfully in various fields (Chehami et al., 2014; Davy et al., 2016; Roux et al., 2004; Sabra et al., 2007, 2008; Shapiro and Campillo, 2004). For structures subject to vibrations, it is then possible to recover data to which classical imaging algorithms can be applied without emitting energy. Furthermore, sensors such as Fiber Bragg Gratings (FBG) on optical fibers, which are unable to emit waves, can be used (Betz et al., 2003). Those sensors have previously been successfully used in combination with piezoelectric transducers (PZT) for active

a) arnaud.recoquillay@cea.fr so data acquisition (Betz et al., 2003; Takeda et al., 2005), and more recently for passive data acquisition using noise cross correlation to recover the active signal first in seismology (Zeng et al., 2017) and then at ultrasonic frequencies for metallic plates (Druet et al., 2018).

The aim of this paper is to present passive guidedwave imaging in composite panels using only Fiber Bragg Gratings. Indeed, as passive methods do not need the emission of ultrasonic waves into the domain, this kind of imaging can be done with classic PZT sensors but also FBGs, opening the way of SHM systems of very low intrusiveness. In a first section is briefly recalled the principle of passive acquisition, before explaining the basis of Fiber Bragg Gratings sensors. Then, two guided wave imaging techniques are presented, namely Delay and Sum and Excitelet before presenting ultrasonic wave measurement using FBGs. In the last section, experimental results obtained with these two algorithms on passive acquisitions for CFRP plates are presented. The first experiment was conducted using PZT sensors to check the feasibility of passive imaging in composite plates, and the full system, using only FBG sensors, is tested in the second experiment.

\section{ACTIVE AND PASSIVE ACQUISITION}

The first step of any imaging algorithm is the data acquisition. For classical guided wave imaging algorithms such as Delay and Sum, this step consists in successively emitting a wave with an actuator and measuring the signal with all other sensors. This method is called, in the following, active acquisition. Another way to obtain 
60 equivalent data is to measure the ambient noise at all sensors simultaneously and then process those signals to115 reconstruct the data used in the imaging algorithm. This method is called, in the following, passive acquisition.

A few algorithms exist to retrieve the data from the 65 ambient noise. The most known, which we will explain in more details and use in the following, is the ambient noise cross-correlation (Lobkis and Weaver, 2001). Another possibility is the passive inverse filter (Gallot et al., 2012), which is well suited when many receivers are used. For example, this technique has been used for guided wave tomography (Druet, 2017; Druet et al., 2019), for which more sensors need to be used to obtain a relevant image of the domain. As the passive inverse filter is based on the inversion of the impulse response matrix, it ${ }^{120}$ 75 is a global process, which reconstructs all active signals from all ambient noise simultaneously. This seems to allow a better reconstruction, in particular if the ambient noise does not have a good distribution in space or frequency domain (Gallot et al., 2012), when many sensors ${ }^{125}$ coda of the noise cross-correlation (Stehly et al., 2008), which may enhance the data's reconstruction from the noise cross-correlation when many sensors are used, by enhancing the spatial distribution of the noise.

In this paper, we focus on the use of the crosscorrelation of the ambient noise as this method has been more widely used until now and works well for any number of sensors. Its principle and hypotheses are recalled in the following. For more details, the reader may refer $^{135}$ 2001: Roux et al., 2005). As the aim of this paper is the ultrasonic imaging of plates, we restrict ourselves to a domain in which linear elastic waves propagate. To the author's best knowledge, the method for elastic waves has ${ }^{140}$ not yet been fully justified theoretically. Hence the justification is given for scalar acoustic waves. Suppose that the ambient noise $\varphi$ is a space-time stationary random field that is also delta correlated in space, which means that, for all $t, \tau>0$ and points $x$ and $y$, the following ${ }^{145}$ relation holds

$$
\langle\varphi(x, t) \varphi(y, \tau)\rangle=K(x) \delta(x-y) F(t-\tau),
$$

where $\langle\cdot\rangle$ denotes the ensemble average, $K$ characterizes150 the spatial support of the sources and $F$ is the normalized time correlation function, its Fourier transform being linked to the spectral energy density of the ambient noise. We suppose in the following that the sources are "well distributed" in space which means, depending on155 the nature of the domain $\Omega$, that $K \equiv 1$ over a sufficient sub-domain (for more details, see (Garnier and Papanicolaou, 2009)).

Let us now consider two sensors, at points $x$ and $y$ acquiring the ambient noise within the time interval 160 $[0, T], T>0$. Those signals are denoted $V_{x}$ and $V_{y}$. The cross-correlation $C$ of these two signals is then

$$
C(x, y, t ; T)=\frac{1}{T} \int_{0}^{T} V_{x}(\tau) V_{y}(\tau+t) \mathrm{d} \tau .
$$

Let $H_{x y}$ be the impulse response of the system formed of the finite domain, the sensor at $x$ acting as a source and the sensor at $y$ acting as a receiver. Suppose that the noise sources have a good distribution in space, that is $K \equiv 1$, then

$$
\lim _{T \rightarrow+\infty} \frac{\mathrm{d} C}{\mathrm{~d} t}(x, y, t ; T) \propto F *\left[H_{x y}(\cdot)-H_{x y}(-\cdot)\right](t)
$$

where $*$ denotes the convolution operation in time and . indicates the variable over which the operator, here the convolution one, is taken. The convolution in time with $F$ in (3) denotes the influence of the spectral density of the ambient noise: the impulse response's reconstruction strongly depends on the time correlation of the noise, and so on the spectral energy density of the ambient noise.

In the following, as elastic waves propagate in the domain of interest, we make the assumption that (3) is still relevant in this case. On a practical point of view, the quality of the data reconstruction will depend on a few parameters: first, the acquisition time $T$ should be taken large enough so that the cross-correlation converges. As will be seen in our applications, acquisition times of the order of at least one second should be used. The spatial repartition of the noise is also important: if the noise is not propagating in the axis going from $x$ to $y$, then the two signals $V_{x}$ and $V_{y}$ are not correlated and the reconstruction will be poor. Likewise, if the energy is only propagating from $x$ to $y$ and not from $y$ to $x$, for example if the domain has a low reverberation, then only the causal part of the signal will be correctly reconstructed. As already stressed out, the frequency distribution of the noise is also to take into account, so that the signals' energy is sufficient for the frequencies of interest. Let us remark that to obtain (3), the assumption has been made that the transfer function of the sensor acting as a source or as a receiver is the same, which in general is true.

The combination of ambient noise cross-correlation and Fiber Bragg Gratings sensors, described in (Druet et al., 2018), allows for the determination of the pitchcatch response, that is the response of the structure for a given sollicitation at one sensor and measured by another sensor, between two FBG sensors as if one was used as an emitter. This offers, on one hand, all the advantages of purely optical fiber sensing, such as dense wavelength multiplexing of the sensing points, immunity to and no generation of Electro-Magnetic Interferences, compatibility with harsh environments such as those involving high or low (cryogenic) temperatures, shock waves (detonic) and also ionizing radiations, as well as the possibility to drastically reduce the intrusiveness of the instrumentation of any kind of structure thanks to reduced wirings. On the other hand, guided elastic waves offer a fine diagnosis of the structure by enabling the detection, localization and sizing of defects as well as a full structure coverage. 


\section{GUIDED WAVE IMAGING TECHNIQUES}

Many existing guided-wave based techniques could be used in combination with passive acquisitions. The only restrictions are the poor control over the reconpending on the application, the frequency range for which the noise has enough energy. In this paper, two classical methods are tested, namely Delay and Sum (DAS) (Michaels, 2008) and Excitelet (Quaegebeur et al., 2011), acquisitions (Kulakovskyi et al., 2019). Both algorithms were considered as they are representative of guided wave imaging techniques, using simple (DAS) or complex (Excitelet) models. Their principle is briefly recalled hereafter.

In both cases, the algorithm is used for detecting a single defect in the plate. Suppose that reference data ${ }_{230}$ were acquired in a sound state, denoted $s_{k}^{\text {ref }}$ for the signal corresponding to the $k$-th emitter-receiver pair, tations of (3), where $H_{x_{k} y_{k}}^{\text {ref }}$ is the impulse response of the reference system for a source at $x_{k}$ and a receiver at $y_{k^{235}}$ and $C$ is a filter that can be added in the post-processing of the reconstructed signals. The signals corresponding to the state at the time of inspection are denoted $s_{k}^{\text {insp }}$. Residual signals are computed by subtracting $s_{k}^{\text {ref }}$ to $s_{k}^{\text {insp }}$ for each emitter-receiver pair. Then, in the case of DAS, the idea is, for each point $x$ of the inspected area, to affect the value of the envelope of the residual signal corinterest going from the emitter to the point $x$ and then to the receiver. Indeed, if a defect is at this point $x$ and the mode is not completely converted into another one, then a wave should be measured by the receiver at this time. By summing over all pairs, the coherence of the data is verified and the points at which there is a higher prob- ${ }^{245}$ ability of presence of the defect are highlighted. More precisely, the Delay and Sum algorithm is the following:

1. Computation of the envelope of the residual signal for each pair:

$$
\tilde{s}_{k}^{\text {res }}(t)=\left|\mathcal{H}\left(s_{k}^{\text {insp }}(t)-s_{k}^{\text {ref }}(t)\right)\right|,
$$

where $\mathcal{H}$ denotes the Hilbert transform.

2. For each point $x$ of the inspected area, computation of the corresponding time of flight for each pair:

$$
T_{k}(x)=\frac{\left|x-x_{k}^{e}\right|}{c_{g}\left(\theta\left(x_{k}^{e}, x\right)\right)}+\frac{\left|x_{k}^{r}-x\right|}{c_{g}\left(\theta\left(x, x_{k}^{r}\right)\right)},
$$

where $x_{k}^{e}(x)$, respectively $x_{k}^{r}(x)$, denotes the posi-255 tion of the emitter, respectively the receiver, for the $k$-th signal, $\theta(x, y)$ is the angle formed by $y-x$ and the positive horizontal axis and $c_{g}$ denotes the energy velocity of the mode of interest, which will be discussed in the following.

3. Extraction of the value of the envelope corresponding to the time of flight and summation over all signals:

$$
P_{\mathrm{DAS}}(x)=\sum_{k=0}^{N-1} \tilde{s}_{k}^{\mathrm{res}}\left(T_{k}(x)\right) .
$$

The strong hypothesis, and the main weakness of this method, is that it considers one single mode, propagating at a fixed energy velocity. It means that no mode conversion by the defect is taken into account, and that other emitted modes may perturb the imaging process. Furthermore, it is well known that guided waves have a dispersive behavior, which means that their energy velocity depends on the frequency. To use this method, it is then recommended to consider a frequency range for which the dispersion of the mode of interest is low, or to consider a narrow-band excitation, which will reduce the resolution as the support in time of the signal will be large.

The Excitelet algorithm is based on the same ideas but compares, thanks to a correlation, the residual signal to the theoretical one obtained when propagating the wave obtained for a given source from the emitter to the considered point and then to the receiver. More precisely, the steps of the algorithm are the following:

1. Computation of the residual signal for each pair:

$$
s_{k}^{\mathrm{res}}(t)=s_{k}^{\mathrm{insp}}(t)-s_{k}^{\mathrm{ref}}(t) .
$$

2. Computation of the theoretical signal for each pair: as the defect is considered as a point scatterer, the theoretical field scattered by this point is equal, in the frequency regime, to the Green's function of the plate time the incident field emitted by the source:

$$
s_{k}^{\mathrm{th}}(t)=\mathcal{F}^{-1}\left[V_{k}^{r}(x, \cdot) V_{k}^{e}(x, \cdot)\right](t),
$$

where $\mathcal{F}^{-1}$ is the inverse Fourier Transform, and $V_{k}^{e}$ and $V_{k}^{r}$ are respectively the wave emitted by the emitter and measured by the receiver of the $k$-th pair in the frequency regime. More precisely,

$$
\begin{array}{r}
V_{k}^{e}(x, \omega)=\int_{\mathbb{R}^{2}} G(x, y, \omega) \boldsymbol{f}(y) \mathrm{d} y U(\omega), \\
V_{k}^{r}(x, \omega)=\int_{\mathbb{R}^{2}} \boldsymbol{g}(y)^{T} G(y, x, \omega) \mathrm{d} y,
\end{array}
$$

$G$ being the Green's function of the problem, $f$ the source function in space, $\boldsymbol{g}$ the measurement function and $U$ the Fourier transform of the excitation signal. Note that $\boldsymbol{f}$ and $\boldsymbol{g}$ are implicitly supposed compactly supported on the support of the sensor, giving meaning to the integrals. Note also that, as the considered problem is the elastodynamic one, $G$ is a matrix whereas $\boldsymbol{f}$ and $\boldsymbol{g}$ are vectors but the signal is scalar in (8).

3. Computation of the correlation between the residual signal and the theoretical one and summation over all signals:

$$
P_{\text {Excitelet }}(x)=\sum_{k=0}^{N} \int_{\mathbb{R}} s_{k}^{\mathrm{res}}(t) s_{k}^{\mathrm{th}}(t) \mathrm{d} t .
$$


algorithms use the same inputs, that is signals at the inspection time and reference ones. Unlike DAS, Excitelet takes into account the guided waves dispersion through the computation of the theoretical signal. How- 320 ever, as the model used is more precise, a good knowl265 edge of the parameters of the sample is mandatory. The use of a correlation in time also implies that the emitted signal should be short to increase the resolution. The computation of the theoretical signal may be time and ${ }_{325}$ memory-consuming in complex cases such as composite materials. To reduce it, a far-field approximation of the Green's function may be used in expressions (10) or only some modal components of the Green's function can be selected. With those two approximations, one obtains 330 $G(x, y, \omega) \approx H_{1}^{(i)}\left(k_{m}(\omega, \theta)|x-y|\right)$, where $H_{1}^{(i)}$ is the Haning on the convention used in the Fourier transform, $k_{m}$ is the wavenumber of the considered mode and $\theta$ is the angle of the vector $x-y$.

Finally, let us point out that, for passive acquisitions, ${ }_{335}$ imposed in post processing, enabling the optimization of the imaging results.

In all the following, the imaging results correspond to normalized indicator functions, that is $P_{a}(x) / \max P_{a}$, where $a$ is either DAS or Excitelet.

\section{ULTRASONIC WAVE MEASUREMENT USING FIBER BRAGG GRATINGS}

Usually employed to measure temperature and strain, Fiber Bragg gratings are increasingly attracting researchers studying ultrasonic guided waves-based imaging methods for SHM applications. Indeed, their low intrusiveness and multiplexing capabilities (both spectrally and temporally) make FBGs an attractive alternative to piezoelectric elements as acoustic receivers. While dense wavelength multiplexing of tens of FBGs on a single optical fiber is commonly used in Telecommunications and even in Sensing applications, its immediate transposition to the case of FBGs used as acoustic receivers is still challenging. Active or passive acquisitions of ultrasonic signals using FBGs sensors require both a sampling frequency ranging from $\mathrm{kHz}$ to $\mathrm{MHz}$ levels while preserving their multiplexing capabilities and a high sensitivity to the strain variations induced by the ultrasonic signals propagating across composite plates. In this study, we have developed a monitoring system dedicated to wavelength-multiplexed FBG used as acoustic receivers. The principle of this measurement system relies on an edge filtering technique. The basic idea of the technique is the use of a tunable laser source per FBG acoustic For a given Bragr wavelength, the lasing wavelength emitted by an external cavity wavelength-tunable laser source is adjusted to the midpoint of one edge of the Bragg peak. Any shift of the Bragg wavelength will modulate the transmitted/reflected optical power.

315 This demodulation scheme is commonly referred to as "edge filtering" technique (Melle et al., 1992). Hence the sampling rate at which each FBG is demodulated does not depend on the acquisition speed of a given spectral analyzer but rather simply on the band pass of both the pigtailed photodetectors and the associated transimpedance electronic. This straightforward demodulation method allows adjusting easily the measurement frequency by simply changing the cutoff frequency of the transimpedance electronic but at the expense of the rather low degree of integration due to the need of one tunable laser source per FBG acoustic receiver. Multiplexing the FBG acoustic receivers simply requires to cascade several tunable lasers and to inject their optical signals to the fiber containing the sensors thanks to an allfiber wavelength multiplexer. A similar component positioned before the photodetectors separates the signals reflected by each FBG. In this study, a transimpedance circuit with cutoff frequencies of $1 \mathrm{MHz}$ has been realized in order to handle four photodetectors simultaneously.

\section{EXPERIMENTAL RESULTS}

\section{A. Passive acquisition using piezoelectric transducers}

A first experiment was carried out using classic piezoelectric transducers of diameter $18 \mathrm{~mm}$ in order to check the feasibility of using passive acquisitions to image composite plates. The used sample was a $1000 \times 600 \times 5.75$ $\mathrm{mm}^{3}$ CFRP plate having 21 plies, all oriented in the same direction. Its material parameters are given in table I.

Table I. Material parameters of the CFRP plates

\begin{tabular}{|c|c|c|c|c|c|c|}
\hline \hline $\begin{array}{c}E_{1}=E_{2} \\
(\mathrm{MPa})\end{array}$ & $\begin{array}{c}E_{3} \\
(\mathrm{MPa})\end{array}$ & $\nu_{12}$ & $\nu_{13}=\nu_{23}$ & $\begin{array}{c}G_{12} \\
(\mathrm{MPa})\end{array}$ & $\begin{array}{c}G_{13}=G_{23} \\
(\mathrm{MPa})\end{array}$ & $\begin{array}{c}\rho \\
\left(\mathrm{kg} \cdot \mathrm{m}^{-3}\right)\end{array}$ \\
\hline 65700 & 4500 & 0.03 & 0.3 & 5100 & 3750 & 1550 \\
\hline \hline
\end{tabular}

The ambient noise was created thanks to a compressed air flow moved randomly over the whole surface of the plate during the acquisition. Eight piezoelectric transducers were placed on a circle of radius $200 \mathrm{~mm}$. This radius was chosen so that reflections on the border of the plate are measured after an incident wave coming from the opposite sensor for frequencies around $20 \mathrm{kHz}$, the measured ambient noise having a significant spectral energy density between $10 \mathrm{kHz}$ and $80 \mathrm{kHz}$. The ambient noise was measured simultaneously on the eight sensors for 10 seconds at a sampling rate of $1 \mathrm{MHz}$. As already mentioned in section III, the quality of the image obtained with DAS or Excitelet is strongly linked to the excitation signal. Here the signal reconstructed from the ambient noise cross-correlation has been filtered to obtain signal characteristics appropriate for those imaging algorithms. More precisely, two different filters were tested: a Hann filter of central frequency $20 \mathrm{kHz}$ and of bandwidth $20 \mathrm{kHz}$ and one of central frequency $45 \mathrm{kHz}$ and of bandwidth $70 \mathrm{kHz}$. For both algorithms, only the $A_{0}$ mode was considered and, in the case of DAS, the 


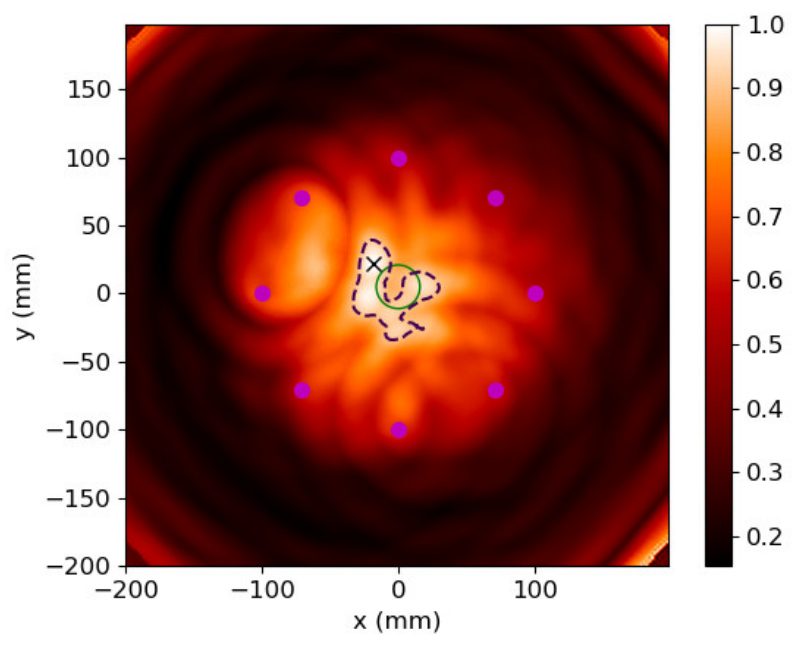

(a) first defect

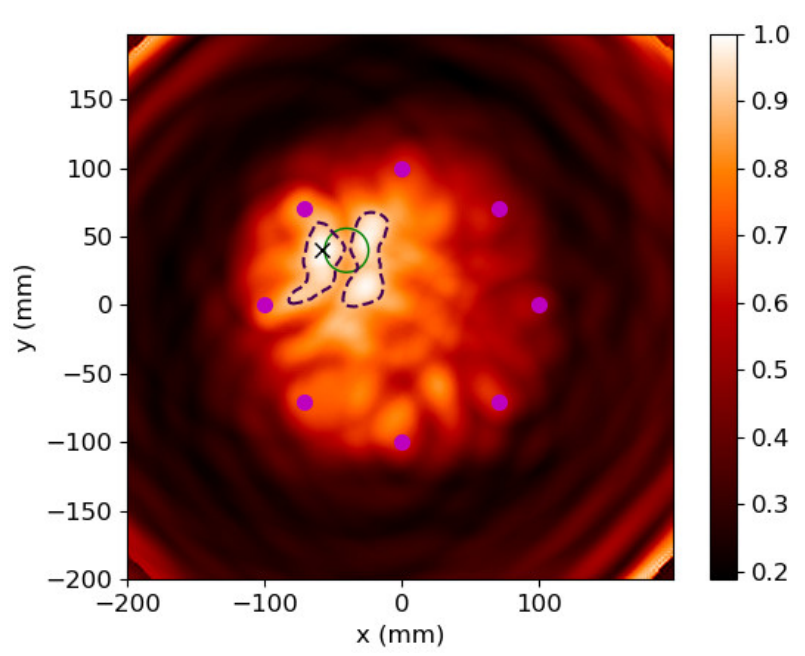

(b) second defect

Figure 1. (color online) DAS applied to passive acquisitions with PZT, frequency range: $10-30 \mathrm{kHz}$.

energy velocity is taken at the central frequency of the considered filter. Synthetic defects were obtained using ${ }^{380}$ a pair of magnets of diameter $32 \mathrm{~mm}$, placed for the first defect at $(0,5)$, which is a slightly off-centered position, and at $(-40,40)$ for the second one. In all the following images, the black dashed lines are contour lines correo.9, added as an illustration. The green circle is the true shape of the defect whereas the black cross is the location of the maximum amplitude of the cartography. First, the imaging results for DAS are shown in figures 1 and 2.

In both cases, the localizations of the defect are well 390 retrieved. It should be noted that the image has a better accuracy in figure 2 than in figure 1, that is the high amplitude spot is smaller in figure 2, enabling a better local-

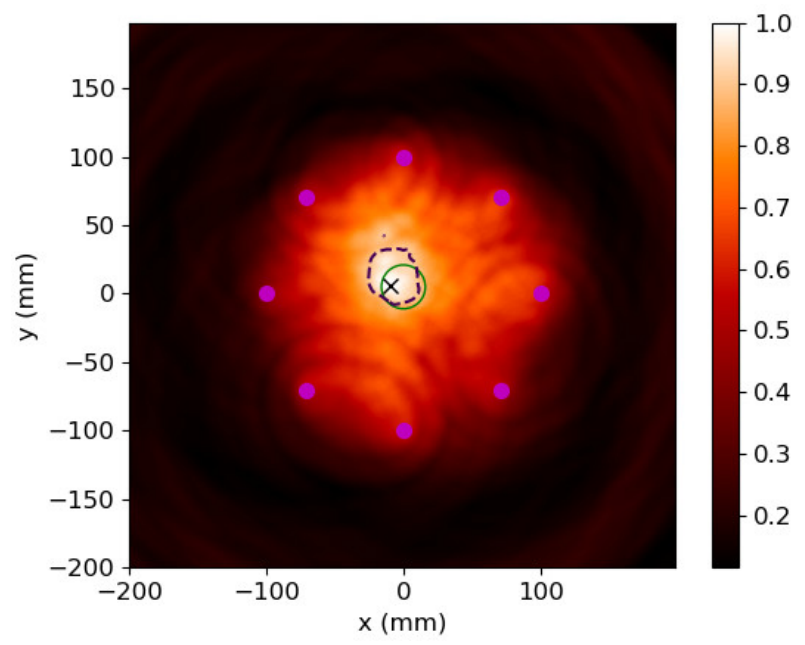

(a) first defect

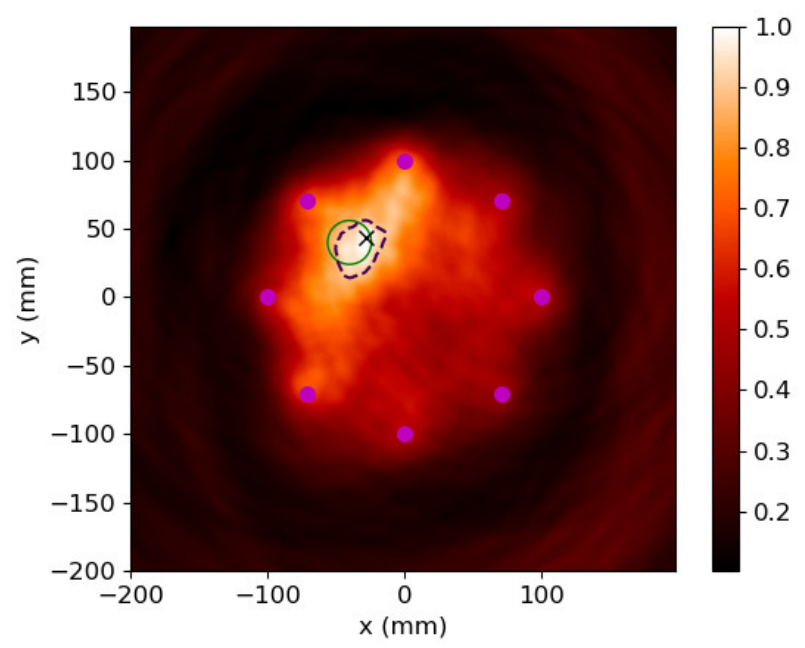

(b) second defect

Figure 2. (color online) DAS applied to passive acquisitions with PZT, frequency range: $10-80 \mathrm{kHz}$.

ization of the defect. This comes from the low dispersion of the $A_{0}$ mode in this frequency range, inducing a better resolution for a bigger bandwidth as the wavepacket is shorter in time.

The imaging results corresponding to Excitelet are presented figures 3 and 4 . The localization is again of good quality, even though the 0.9 contour is wider in this case. This may come from a greater sensitiveness to the noise in the passive reconstruction: as will be seen in $\mathrm{VB}$, Excitelet is more precise as long as the data are of good quality. Finally, in table II is given the distance between the center of the defect and the maximum of the cartography. Except for the case of low frequency DAS, the distance is lower than the radius of the defect, 


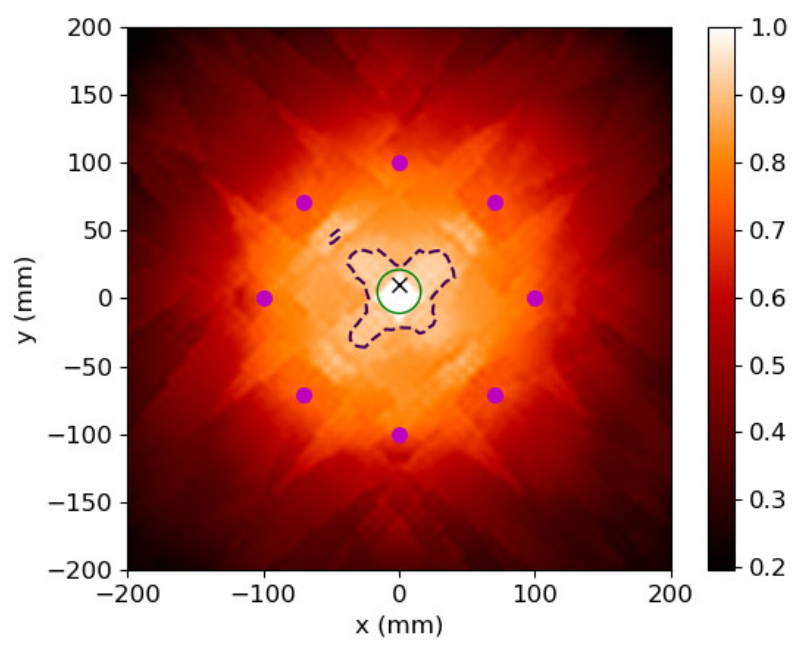

(a) first defect

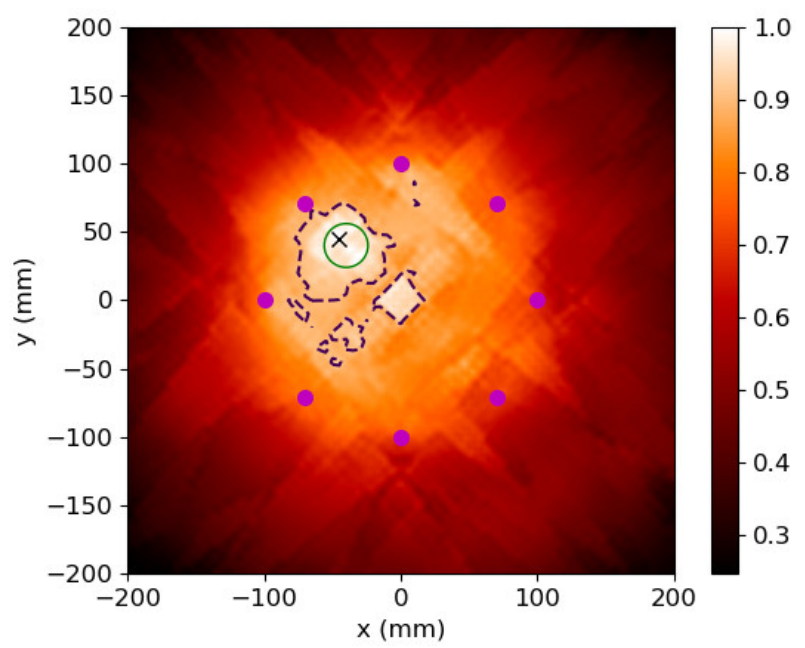

(b) second defect

Figure 3. (color online) Excitelet applied to passive acquisitions with PZT, frequency range: $10-30 \mathrm{kHz}$.

which is the best result possible given the point scatterer

\section{B. Passive acquisition using Fiber Bragg Gratings on optical fibers}

The acquisition using FBG sensors was done on a415 $1010 \times 610 \times 2.2 \mathrm{~mm}^{3} \mathrm{CFRP}$ plate having 8 plies, all oriented in the same direction. Its material parameters are the same as the previous plate, given in table I. Eight FBG sensors were placed on the surface of the plate on a circle of radius $150 \mathrm{~mm}$ centered at the middle of the 420 plate. Due to the used acquisition device, the signals of only four FBG could be recorded simultaneously. As the ambient noise needs to be recorded simultaneously

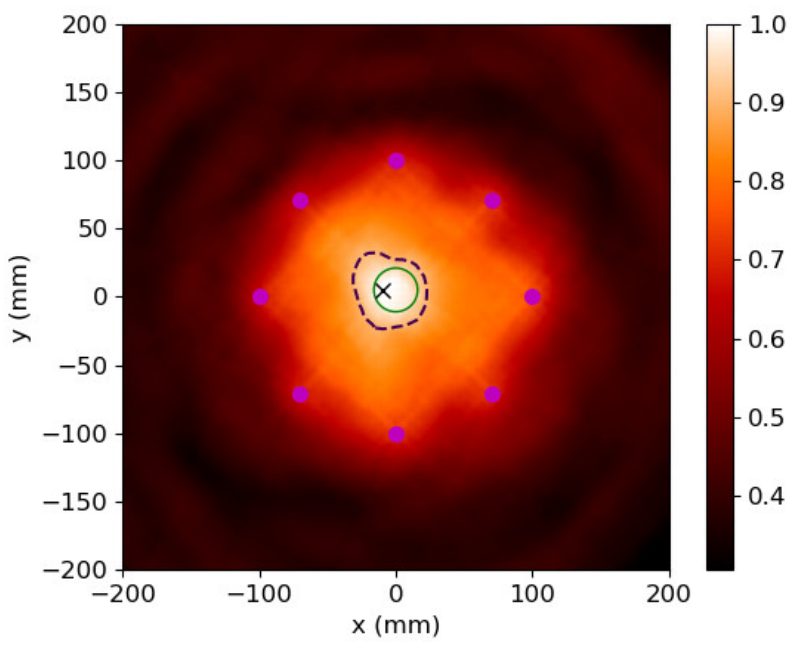

(a) first defect

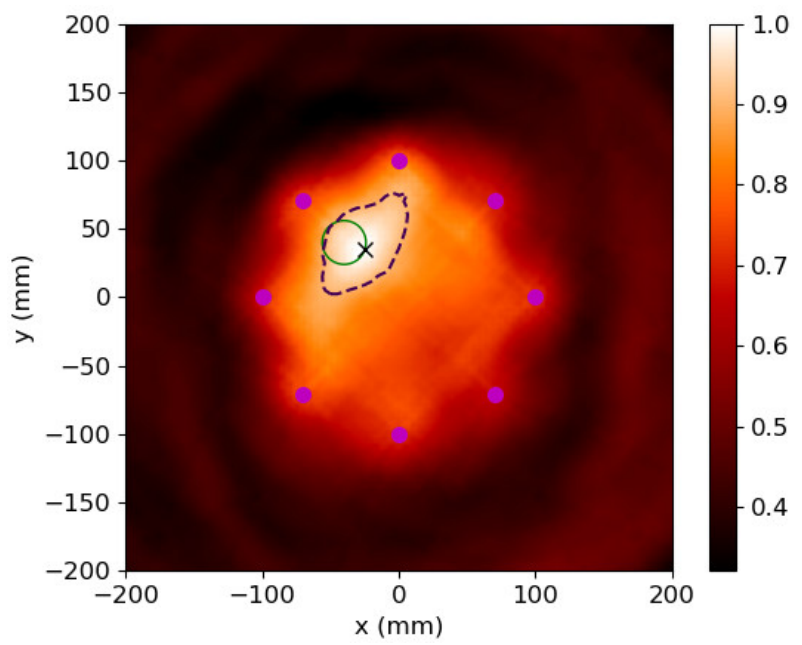

(b) second defect

Figure 4. (color online) Excitelet applied to passive acquisitions with PZT, frequency range: $10-80 \mathrm{kHz}$.

on the sensors to recover the impulse response from the cross-correlation, this means that only a part of the data was obtained (see figure 5). Only 24 different signals can then be reconstructed, where 56 different pairs exist and were used in the previous section. Note that all FBGs are oriented toward the center of the circle in order to maximize their sensitivity in the region of interest (Betz et al., 2003). Defects were again simulated thanks to a pair of magnets of diameter $32 \mathrm{~mm}$, placed at $(-44,82)$ for the first position and $(-32,-24)$ for the second one. The noise was measured during 50 seconds as to ensure a good reconstruction of the signals. The Fiber Bragg Gratings are $5 \mathrm{~mm}$ long with a reflectivity greater than 97\%. They were photowritten in a polyimide-coated ger- 
Table II. Distance of the maximum to the center of the defect, PZT case

\begin{tabular}{|l|c|c|c|}
\hline \hline Algorithm & case & $\begin{array}{c}\text { wavelength } \\
(\mathrm{mm})\end{array}$ & $\begin{array}{c}\text { distance } \\
(\mathrm{mm})\end{array}$ \\
\hline DAS & low frequency, defect 1 & 31 & 25 \\
& low frequency, defect 2 & 31 & 18 \\
& high frequency, defect 1 & 20 & 10 \\
& high frequency, defect 2 & 20 & 13 \\
\hline Excitelet & low frequency, defect 1 & 31 & 5 \\
& low frequency, defect 2 & 31 & 7 \\
& high frequency, defect 1 & 20 & 10 \\
& high frequency, defect 2 & 20 & 16 \\
\hline \hline
\end{tabular}

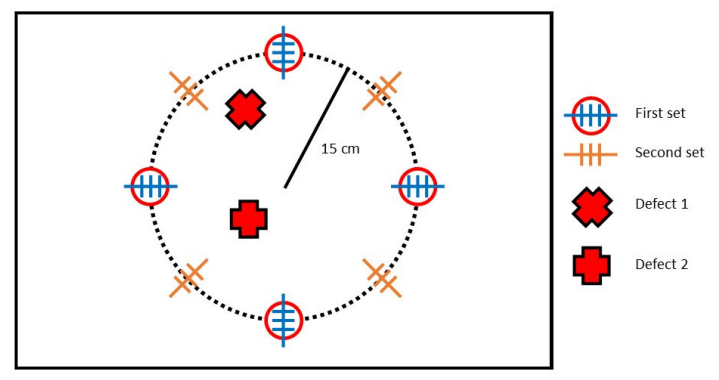

Figure 5. Scheme of the acquisition setup using FBGs

manosilicate singlemode optical fiber using a $\mathrm{KrF}$ laser emitting at $248 \mathrm{~nm}$ and a Talbot interferometer.

The images obtained using DAS are presented figures 6 and 7. In this case, the obtained images do not enable to recover the position of the defects. An approximate position of the first defect may be recovered for both bandwidth, but the second one is not retrieved. In both cases, a strong noise exists in the images. Let us recall that less than half the data used in the previous section were available in this case, which may explain for a great part the poor results.

Applying Excitelet, figures 8 and 9, the results are $_{450}$ more satisfying: even though there still is noise in the images, the locations of the defect are properly reconstructed, with a better accuracy for the central frequency of $45 \mathrm{kHz}$ as expected. Indeed, in figure 8, the algorithm detects the presence of a defect but its position and size ${ }_{455}$ of the possibility of using FBG sensors with a passive acquisition for guided wave imaging of composite panels. The imaging results obtained with both algorithms are of the same quality using FBG or PZT, even though ${ }_{460}$ acquired with the FBGs. It is interesting to note that both algorithms may be used with passive data as DAS

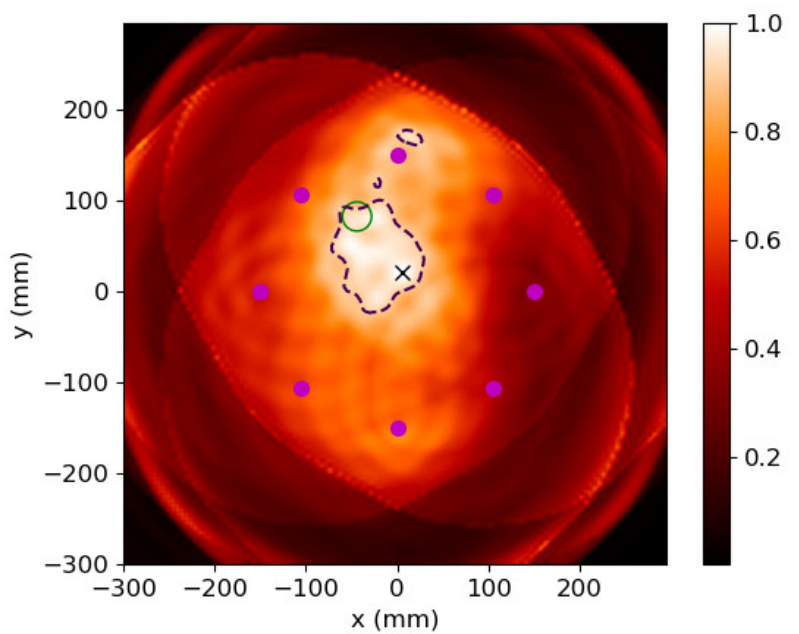

(a) first defect

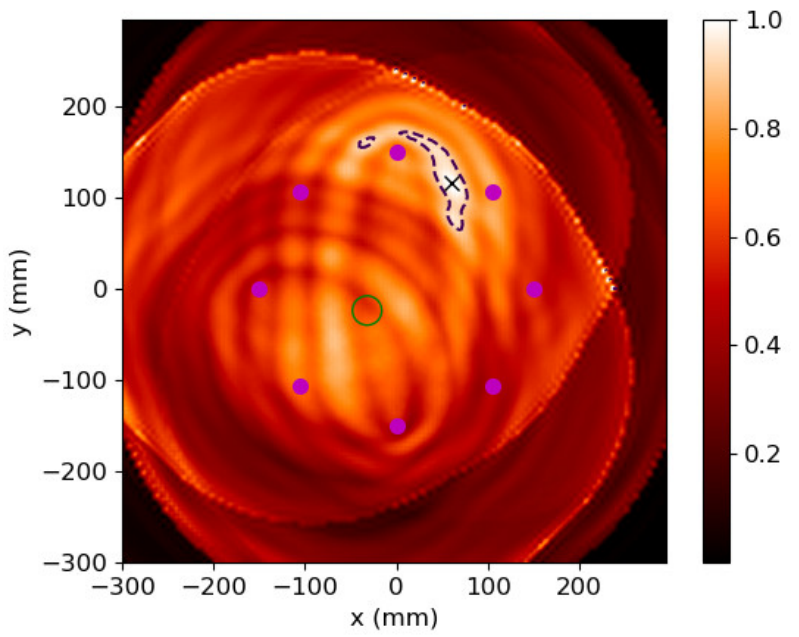

(b) second defect

Figure 6. (color online) DAS applied to passive acquisitions with $\mathrm{FBG}$, frequency range: $10-30 \mathrm{kHz}$.

is easy to use and does not require a high knowledge of the wave propagation in the plate whereas Excitelet requires a higher knowledge leading to a higher accuracy. Once again, the distance between the center of the defect and the maximum of the cartography is summarized in table III. In this case, as expected, the maximum is in general far from the defect. It should nevertheless be noted that the results are generally better for the high frequency filter, which is expected, and that the results ar particularly good in this case for Excitelet.

Before concluding, the imaging results of Excitelet are plotted in figure 10 for various noise acquisition time. It can be seen that the image's resolution increases with the acquisition duration due to the convergence of the cross-correlation of the ambient noise. It seems that, 


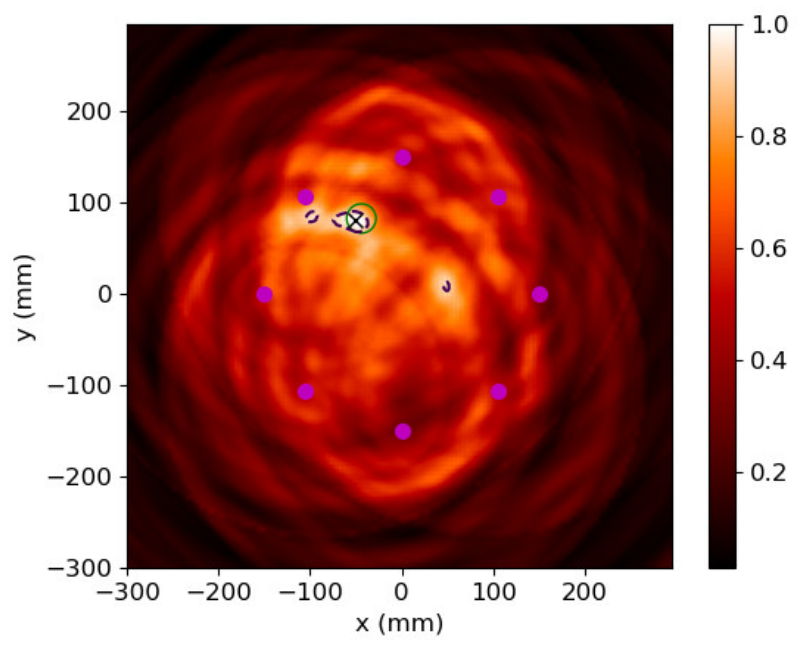

(a) first defect

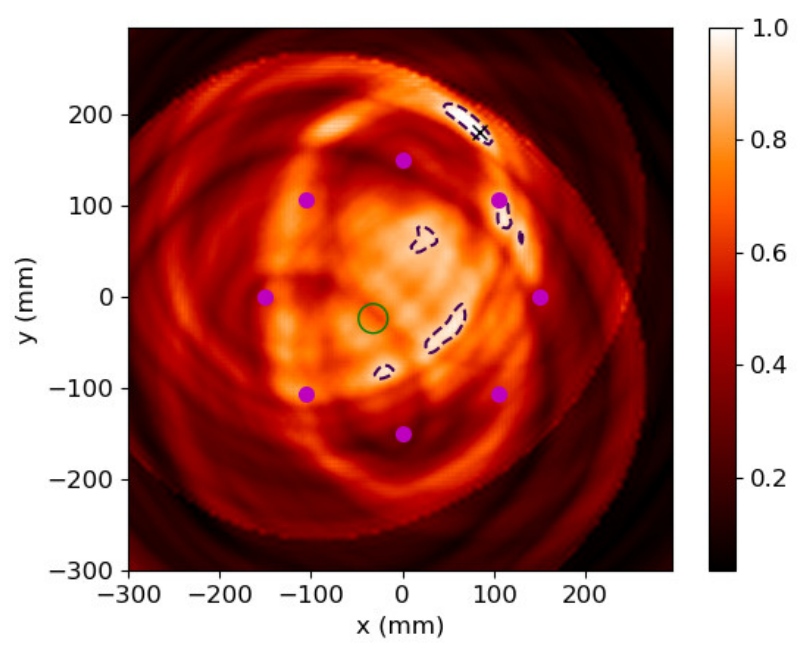

(b) second defect

Figure 7. (color online) DAS applied to passive acquisitions with $\mathrm{FBG}$, frequency range: $10-80 \mathrm{kHz}$.

with the current system, an ambient noise acquisition of 30 seconds is necessary to have a sufficient signal reconstruction, enabling the localization of a defect. But ${ }^{480}$ this value depends on various factors, among which are: the frequency content of the noise source, the frequency dependent transfer function of the sensors and of the acquisition system. For example, stronger analogical filtering of the noise around the bandwidth of interest, or ${ }^{48}$ equivalently a noise with a bandwidth closer to the one of interest, can help to have a better resolution in the reconstruction. As the noise source depends strongly on the application and is a parameter on which the user has no control, tailoring the acquisition system towards the ${ }^{490}$ expected frequency content of the noise may be a satisfying solution.

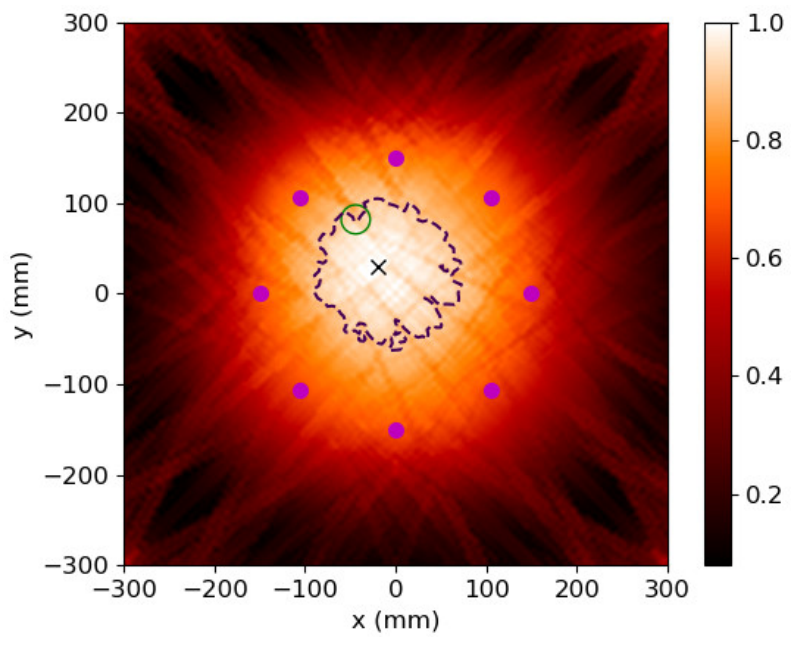

(a) first defect

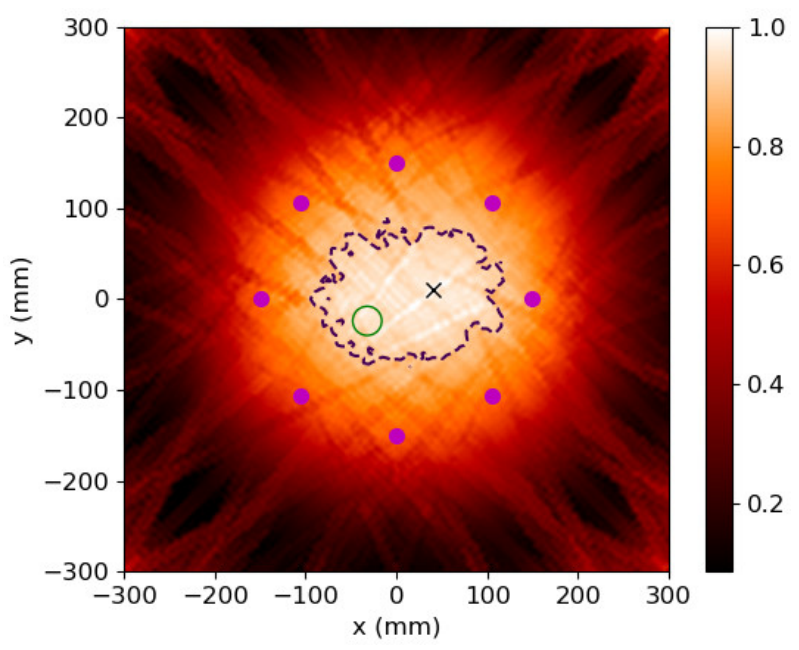

(b) second defect

Figure 8. (color online) Excitelet applied to passive acquisitions with FBG, frequency range: $10-30 \mathrm{kHz}$.

\section{CONCLUSION}

In this article has been shown the feasibility to image composite CFRP plate with elastic guided wave imaging algorithms on passive acquisitions thanks to FBG sensors. Many parameters need to be thoroughly studied to determine in which configurations this approach can be used and several ways of improvement are being investigated: first of all, not all signals could be reconstructed with the current acquisition system, limited to only 4 parallel FBG channels. The development of a system with more channels is undergoing at CEA and will lead to improved imaging results in the future. Another parameter is the angular sensitivity of the FBG sensors: the measurement depends on the angle between the incident wave on the gratings and its direction. Even though 


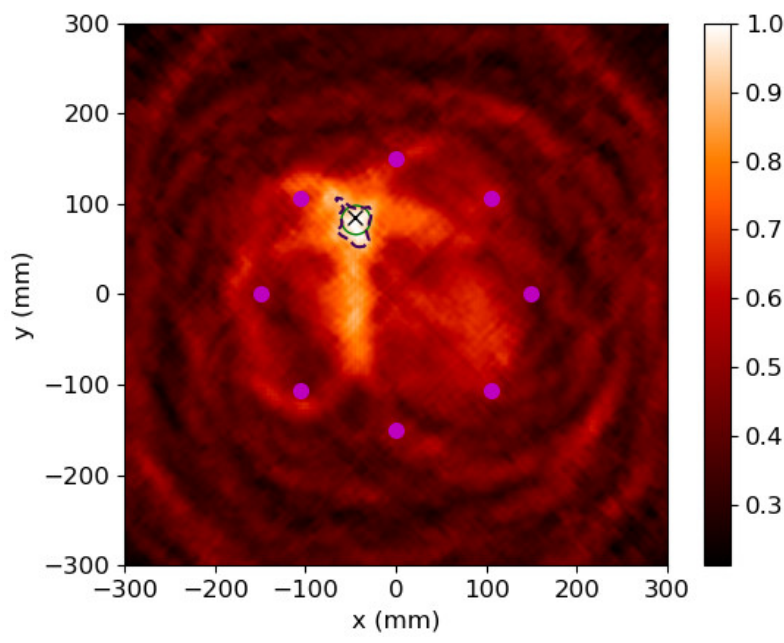

(a) first defect

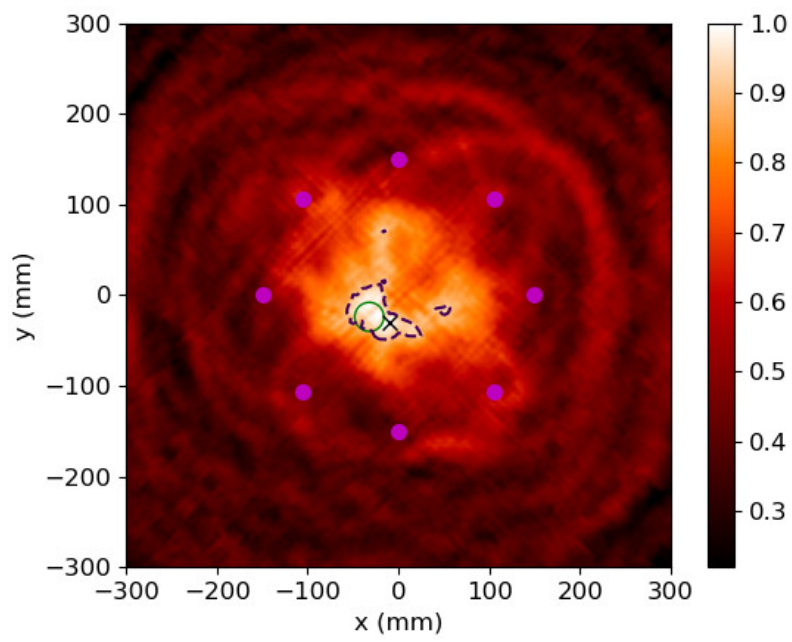

(b) second defect

Figure 9. (color online) Excitelet applied to passive acquisitions with FBG, frequency range: $10-80 \mathrm{kHz}$.

Table III. Distance of the maximum to the center of the defect, FBG case

\begin{tabular}{|l|c|c|c|}
\hline \hline Algorithm & case & $\begin{array}{c}\text { wavelength } \\
(\mathrm{mm})\end{array}$ & $\begin{array}{c}\text { distance } \\
(\mathrm{mm})\end{array}$ \\
\hline DAS & low frequency, defect 1 & 31 & 79 \\
& low frequency, defect 2 & 31 & 167 \\
& high frequency, defect 1 & 20 & 6 \\
& high frequency, defect 2 & 20 & 235 \\
\hline Excitelet & low frequency, defect 1 & 31 & 57 \\
& low frequency, defect 2 & 31 & 80 \\
& high frequency, defect 1 & 20 & 3 \\
& high frequency, defect 2 & 20 & 23 \\
\hline \hline
\end{tabular}

tages of the FBGs, such as their high temperature or radiation resistance. (2003). "Acousto-ultrasonic sensing using fiber Bragg gratings," Smart Materials and Structures 12(1), 122.

Chehami, L., Moulin, E., De Rosny, J., Prada, C., Bou Matar, O., Benmeddour, F., and Assaad, J. (2014). "Detection and localization of a defect in a reverberant plate using acoustic field correlation," Journal of applied Physics 115(10), 104901.

Davy, M., De Rosny, J., and Besnier, P. (2016). "Green's function retrieval with absorbing probes in reverberating cavities," Physical review letters 116(21), 213902.

Druet, T. (2017). "Tomographie passive par ondes guidées pour des applications de contrôle santé intégré," Ph.D. thesis, Université de Valenciennes et du Hainaut-Cambrésis.

Druet, T., Chapuis, B., Jules, M., Laffont, G., and Moulin, E. (2018). "Passive guided waves measurements using fiber Bragg gratings sensors," The Journal of the Acoustical Society of America 144(3), 1198-1202.

Druet, T., Recoquillay, A., Chapuis, B., and Moulin, E. (2019). "Passive guided wave tomography for structural health monitoring," Journal of the Acoustical Society of America .

Gallot, T., Catheline, S., Roux, P., and Campillo, M. (2012). "A passive inverse filter for Green's function retrieval," The Journal of the Acoustical Society of America 131(1), EL21-EL27.

Garnier, J., and Papanicolaou, G. (2009). "Passive sensor imaging using cross correlations of noisy signals in a scattering medium," SIAM Journal on Imaging Sciences 2(2), 396-437.

Kulakovskyi, A., Mesnil, O., Lhémery, A., Chapuis, B., and d'Almeida, O. (2019). "Defect imaging in layered composite plates and honeycomb sandwich structures using sparse piezoelectric transducers network," 1184(1), 012001.

Lobkis, O. I., and Weaver, R. L. (2001). "On the emergence of the Green's function in the correlations of a diffuse field," The Journal of the Acoustical Society of America 110(6), 3011-3017.

Melle, S. M., Liu, K., and Measures, R. (1992). "A passive wavelength demodulation system for guided-wave bragg grating sensors," IEEE Photonics Technology Letters 4(5), 516-518.

Michaels, J. E. (2008). "Detection, localization and characterization of damage in plates with an in situ array of spatially distributed ultrasonic sensors," Smart Materials and Structures $\mathbf{1 7}(3), 035035$.

Quaegebeur, N., Masson, P., Langlois-Demers, D., and Micheau, P. (2011). "Dispersion-based imaging for structural health monitoring using sparse and compact arrays," Smart Materials and Structures 20(2), 025005.

55 Roux, P., Kuperman, W., and Group, N. (2004). "Extracting coherent wave fronts from acoustic ambient noise in the ocean," 


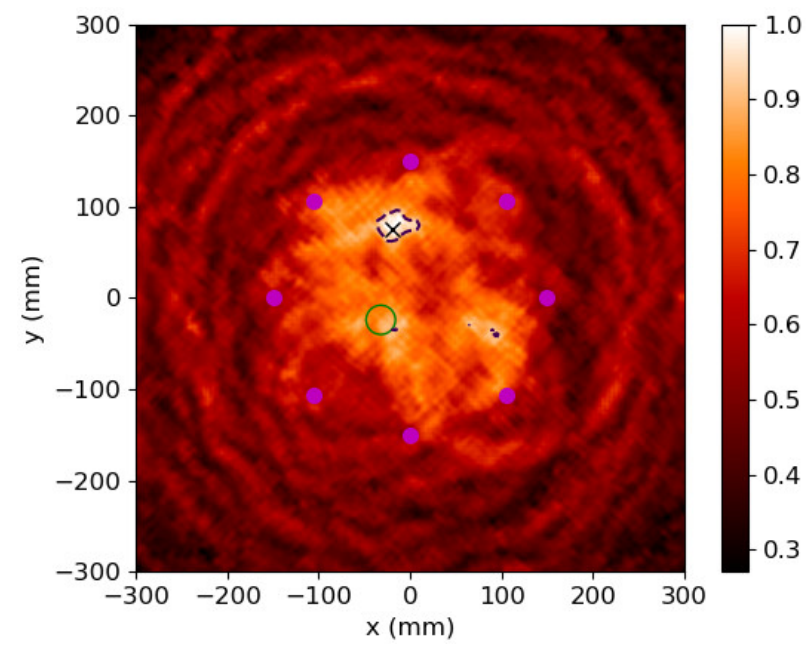

(a) $5 \mathrm{~s}$

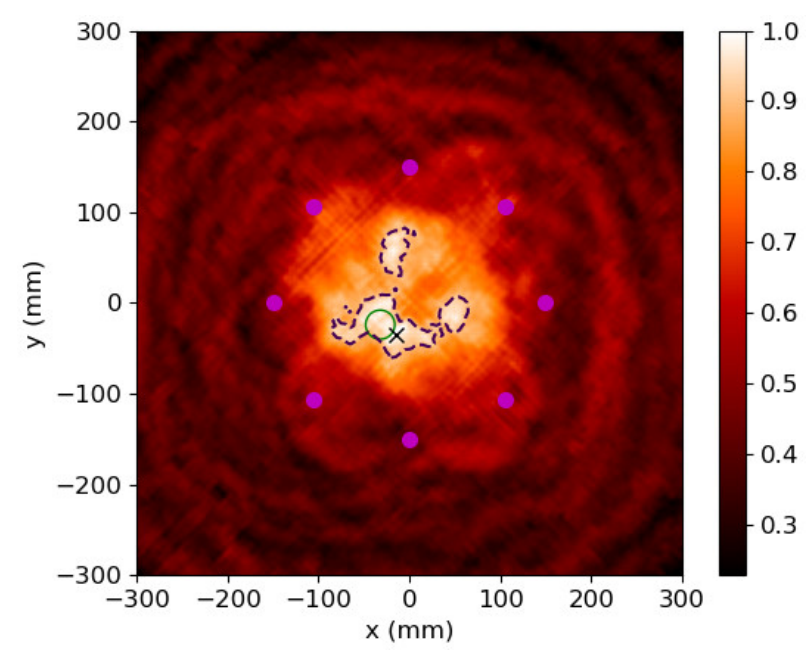

(c) $30 \mathrm{~s}$

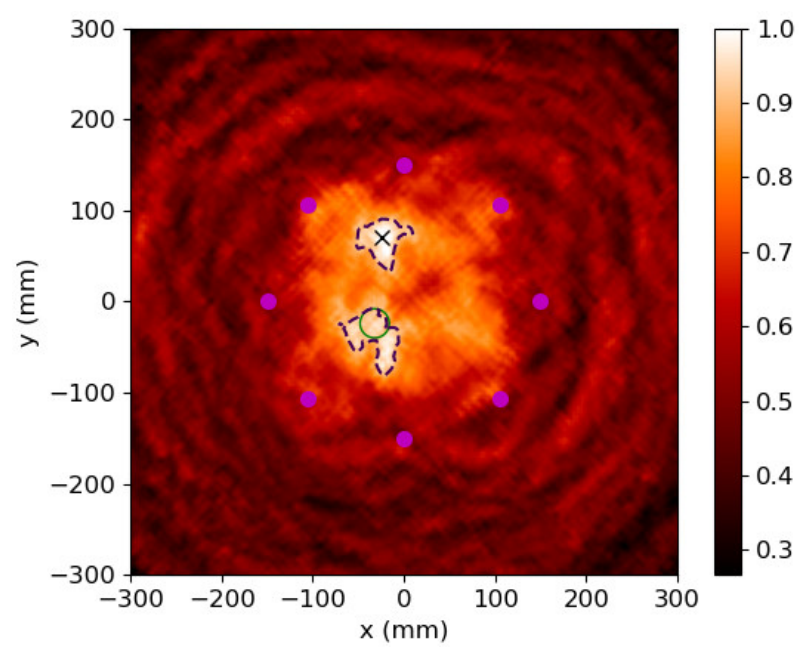

(b) $10 \mathrm{~s}$

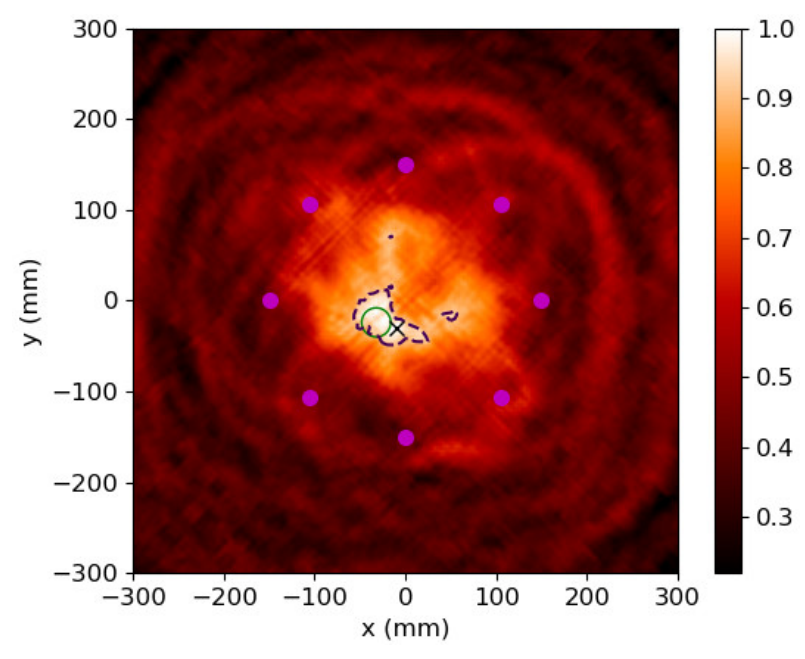

(d) $50 \mathrm{~s}$

Figure 10. (color online) Excitelet applied to reconstructed signals for various noise acquisition durations.

The Journal of the Acoustical Society of America 116(4), 19952003.

Roux, P., Sabra, K. G., Kuperman, W. A., and Roux, A. (2005). "Ambient noise cross correlation in free space: Theoretical ap-580 proach," The Journal of the Acoustical Society of America $\mathbf{1 1 7}(1), 79-84$.

Sabra, K. G., Conti, S., Roux, P., and Kuperman, W. (2007). "Passive in vivo elastography from skeletal muscle noise," Applied physics letters 90(19), 194101.

Sabra, K. G., Srivastava, A., Lanza di Scalea, F., Bartoli, I., Rizzo, P., and Conti, S. (2008). "Structural health monitoring by extraction of coherent guided waves from diffuse fields," The Journal of the Acoustical Society of America 123(1), EL8-EL13.

Shapiro, N. M., and Campillo, M. (2004). "Emergence of broadband Rayleigh waves from correlations of the ambient seismic noise," Geophysical Research Letters 31(7).

Stehly, L., Campillo, M., Froment, B., and Weaver, R. L. (2008). "Reconstructing Green's function by correlation of the coda of the correlation (C3) of ambient seismic noise," Journal of Geophysical Research: Solid Earth 113(B11).
Takeda, N., Okabe, Y., Kuwahara, J., Kojima, S., and Ogisu, T. (2005). "Development of smart composite structures with small-diameter fiber Bragg grating sensors for damage detection: Quantitative evaluation of delamination length in CFRP laminates using Lamb wave sensing," Composites science and technology 65(15-16), 2575-2587.

Zeng, X., Lancelle, C., Thurber, C., Fratta, D., Wang, H., Lord, N., Chalari, A., and Clarke, A. (2017). "Properties of noise crosscorrelation functions obtained from a distributed acoustic sensing array at Garner Valley, California," Bulletin of the Seismological Society of America 107(2), 603-610. 
